\title{
Calculation of minor actinides transmutation in ADS reactors
}

\author{
Patricia Kelly Taipe Torres* \\ Programa de Engenharia Nuclear, COPPE-UFRJ \\ Rio de Janeiro, Brazil \\ E-mail: ptorresecon.ufrj.br
}

\section{Fernando Carvalho da Silva}

Programa de Engenharia Nuclear, COPPE-UFRJ

Rio de Janeiro, Brazil

E-mail: fernando@con.ufrj.br

\section{Antonio Carlos Marques Alvim}

Programa de Engenharia Nuclear, COPPE-UFRJ

Rio de Janeiro, Brazil

E-mail: aalvimegmail.com

\begin{abstract}
One of the points in debate and therefore of mistrust about the use of nuclear energy as a source of electricity generation is what to do with spent fuel elements. These fuel elements have several radionuclides (long-lived fission products and minor actinides) that turns them into a system with very high radiotoxicity. One possibility to solve this issue is to repackage them into suitable geological repositories. Studies are underway to investigate the possibility of incinerating long-lived fission products and transmute minor actinides, that are the main contributors to radiotoxicity, in ADS reactors. The transmutation of minor actinides in ADS reactors greatly diminishes this radiotoxicity. ADS are subcritical reactors guided by a source and transmutation of minor actinides requires high fluxes in the region where these actinides are. Therefore, studies are underway to determine the appropriate intensity of the source and neutron fluxes for a chosen level of subcriticality. In this work we present results of studies done, for a certain subcriticality level, aiming at showing the effectiveness of an ADS reactor to decrease radiotoxicity considering different levels of subcriticality and different source intensities, that is, a sensivity study could be made for this purpose.
\end{abstract}

X Latin American Symposium on Nuclear Physics and Applications (X LASNPA),

1-6 December 2013

Montevideo, Uruguay

\footnotetext{
*Speaker.
} 


\section{Introduction}

At present there are 437 reactors in operation worldwide, with a total generating capacity of 372.1 GWe, of which 257 are Light Water Reactor (LWR) [1]. A LWR typically generates about 20-30 tons of spent fuel per year [2]. Approximately 96.3\% of the mass of spent nuclear fuel is composed of uranium and short-lived fission products, which do not represent the long-term radiological hazard. This mass has $0.85 \%$ of $\mathrm{Pu}, 0.16 \%$ Minor Actinide (MA) (mainly Np, Am and $\mathrm{Cm}$ ) and $2.7 \%$ of Long Lived Fission Product (LLFP) such as Sr90 , Cs137, Tc99 and I129 being the first two isotopes with almost 30 years of half-life, and the last two to more than a million year half-life. Nuclear transmutation is the effective transformation that converts an isotope into another isotope. Being the MA radioactive long-lived, high-level and LLFP, the main contributors of radioactive waste, has been thinking of actinides and fission convert LLFP in stable isotopes or short life [3]. The resulting Pu of transmutation can be reused for subsequent refills MOX fuel or as a material for manufacturing nuclear batteries. A great alternative sustainable solution that is based on this method, is being provided by an Accelerator Driven Systems (ADS), using as an option of reprocessing. Since 1990, the ADS are being developed in order to reduce the radiotoxicity of spent nuclear fuel, energy production, and also as a source of neutrons. Such dedicated cores comes great potential for a significant reduction of the radiotoxicity of actinides, created from the depletion of the nuclear fuel [4]. The ADS consists of a high-energy accelerator capable of producing neutrons from a beam of high-energy particles directed at a target, causing spallation reaction, which arise neutrons. These spallation neutrons are sent to a subcritical core. This keeps the subcritical core stable, due to the fact that it is powered by an external neutron source. Further, the ADS can be used as a neutron source controlled, since it is a system that could be disconnected by simply stopping the beam of high energy particles, instead of having to insert control rods, for example. Reprocessing of spent fuel followed by their geological storage (temporary or direct) or direct storage (without reprocessing) are now provided some solutions. This is of profound knowledge and that is what creates social acceptance issues regarding the use of nuclear energy. The concept of partitioning and transmutation has been pointed out in several studies in the past and more recently in the study of Generation IV reactors as a strategy that can relax the constraints on the geological storage and that can reduce the monitoring period for scales technological and manageable [5]. The theme focuses on which this work is based on the calculation of transmutation of materials that make up the reactor core, but with a clear focus on changes in MA, as these are beyond the LLFP the main suppliers of the radiotoxicity of high-level waste in nuclear, which are generated during operation of the reactor. The topic of nuclear transmutation is of interest in many areas of physics and nuclear applications, to optimize production of nuclides, transmutation of radioactive waste long life and evolution of the reactor cycle.

\section{Methodology}

\subsection{Neutron Diffusion Equation}

We work with the one-dimensional diffusion equation and per two groups. Furthermore, average fluxes were used in the depletion equations and $\bar{\varphi}_{g}^{m}$, will be calculated as follows: First the 
discretization in the time is done, with known initial concentrations. Then one-dimensional nuclear model is spatially discretized into regions. Our method has its starting point in the neutron diffusion equation and Fick's Law, in steady state and time instant $t_{l}$,

$$
\begin{aligned}
\frac{\mathrm{d}}{\mathrm{d} x} J_{g}\left(x, t_{l}\right)+\Sigma_{R g}\left(x, t_{l}\right) \phi_{g}\left(x, t_{l}\right)=\chi_{g} \sum_{g^{\prime}=1}^{2} v \Sigma_{f g^{\prime}}\left(x, t_{l}\right) \phi_{g^{\prime}}\left(x, t_{l}\right)+ \\
\quad+\sum_{\substack{g^{\prime}=1 \\
g^{\prime} \neq}}^{2} \Sigma_{g g^{\prime}}\left(x, t_{l}\right) \phi_{g^{\prime}}\left(x, t_{l}\right)+S_{g}\left(x, t_{l}\right) ; l=1, \ldots, \mathrm{L}
\end{aligned}
$$

and

$$
J_{g}\left(x, t_{l}\right)=-D_{g}\left(x, t_{l}\right) \frac{\mathrm{d} \phi_{g}}{\mathrm{~d} x}\left(x, t_{l}\right) ; \quad g=1,2,
$$

\subsection{Production and Depletion chain}

Two types of nuclides chains were considered,
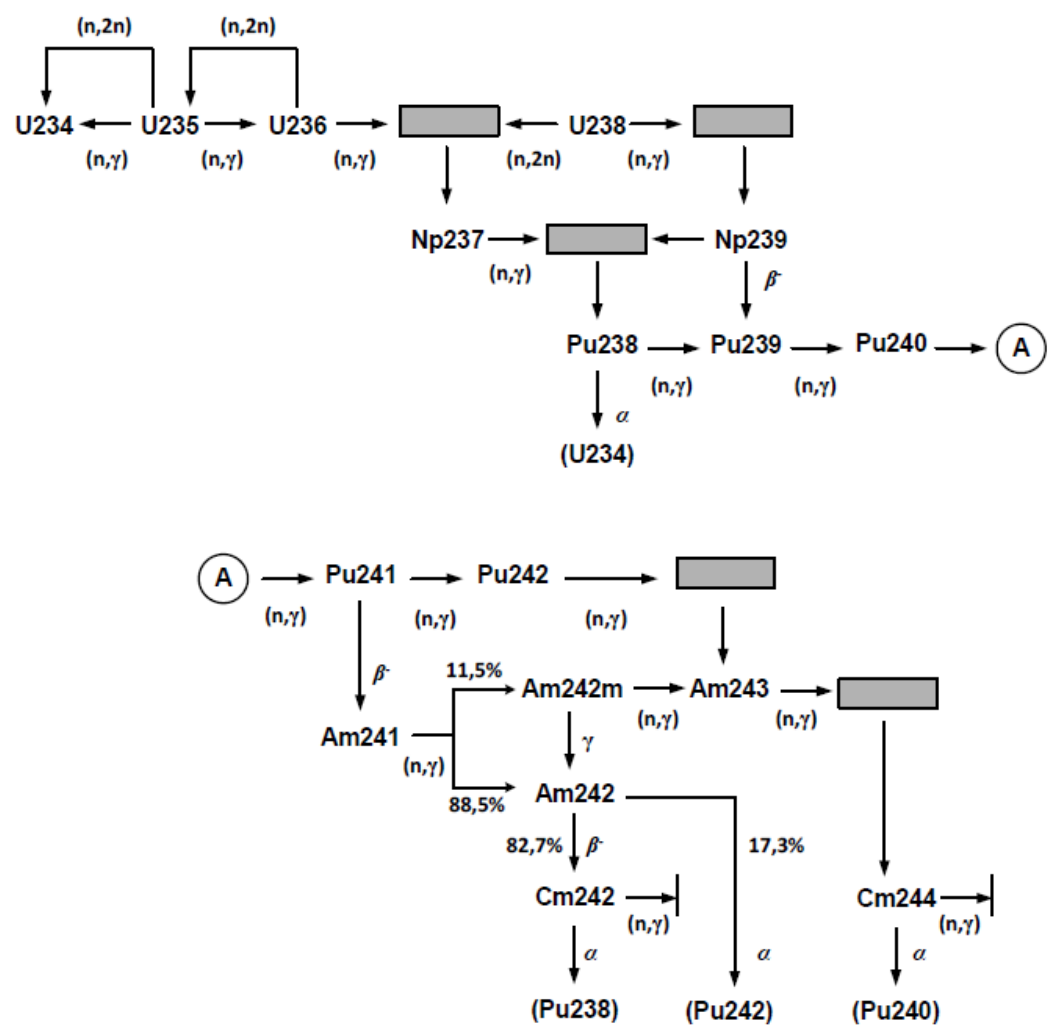

Figure 1: Depletion Chain of Actinides.

\subsection{Depletion Equations and Matrix Form}

These equations are solved in one subroutine called 'Calcula Depleção', and give us the nuclide concentrations in each region $m$, during the step time, $\triangle t_{l}=t_{l}-t_{l-1}$. The depletion equations for actinides and fission products, can be written as follows: 


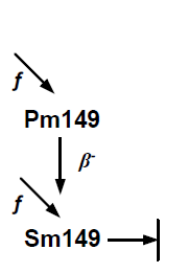

(a)

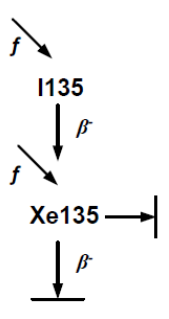

(b)

Figure 2: Depletion Chain of Samarium (a) and Xenonium (b)

$$
\begin{gathered}
\frac{\mathrm{d}}{\mathrm{d} t} N_{i}^{m}(t)=\sum_{j=1}^{I_{f}} e_{i j}^{m}(t) N_{j}^{m}(t) ; i=1, I_{f} \\
e_{i i}^{m}(t)=-\left(\lambda_{i}+\sum_{g=1}^{2} \sigma_{a g}^{i} \bar{\varphi}_{g}^{m}(t)+\sigma_{N, 2 n}^{i} \bar{\varphi}_{1}^{m}(t)\right), \\
e_{i j}^{m}(t)=\alpha_{i}^{j} \lambda_{j} \text { ou } \alpha_{i}^{j} \sum_{g=1}^{2} \sigma_{c g}^{i} \bar{\varphi}_{g}^{m}(t) ; j<i
\end{gathered}
$$

and

$$
e_{i j}^{m}(t)=\lambda_{j} \text { ou } \sigma_{N, 2 n}^{i} \bar{\varphi}_{1}^{m}(t) ; j>i .
$$

being $\lambda$ a representation of the decay constant $(\alpha, \beta$ or $\gamma)$ of the isotope $j, \bar{\varphi}_{g}^{m}$ is the average neutron flux in the region $m$ of the group $g$, the normalized power operation in the burning interval of $\triangle t_{l}$. The constant $\alpha_{i}^{j}$ is the fraction of nuclide $i$ production by decay or by neutron capture in nuclide $j$.

\section{Numerical Method}

The $\mathbf{N}_{i}^{m}\left(t_{l-1}\right)$ is given as a initial condition, and $E_{l}^{m}$ is taken as a constant in the step time $\Delta t_{l}$. Thus the actinide concentrations are:

$$
\frac{\mathrm{d}}{\mathrm{d} t} \mathbf{N}^{m}(t)=E_{l}^{m} \mathbf{N}^{m}(t) ; \quad t_{l-1} \leq t<t_{l} .
$$

This system has formal solution given by [6]

$$
\mathbf{N}^{m}\left(t_{l}\right)=e^{E_{l}^{m} \triangle t_{l}} \mathbf{N}^{m}\left(t_{l}\right), \triangle t_{l}=t_{l}-t_{l-1}
$$

being $\exp \left(E_{l}^{m} \triangle t_{l}\right)$ the transition matrix. Once nuclide concentrations are known for time instant $t_{l-1}$, we use the Transition Matrix Method to know the concentrations subsequent for the time instant $t_{l}$. Finally, it is possible to calculate nuclides concentrations of MA and FP at the desired time. 


\subsection{Calculation Program}

In this section the developed computer program to perform the calculations of transmutation is described. As the program's name itself says, it will do the calculation of MA transmutation. Its task consisted on the recalculation at each instant in time of the nuclear parameters, the average flux by regions and the changes in the nuclide concentrations. This program is based on an evolutive process, which starts with known values of concentrations, average flux and nuclear parameters, that subsequently they will be recalculate in the mentioned subroutines. To have a clear idea of the main program, see figure 3 .

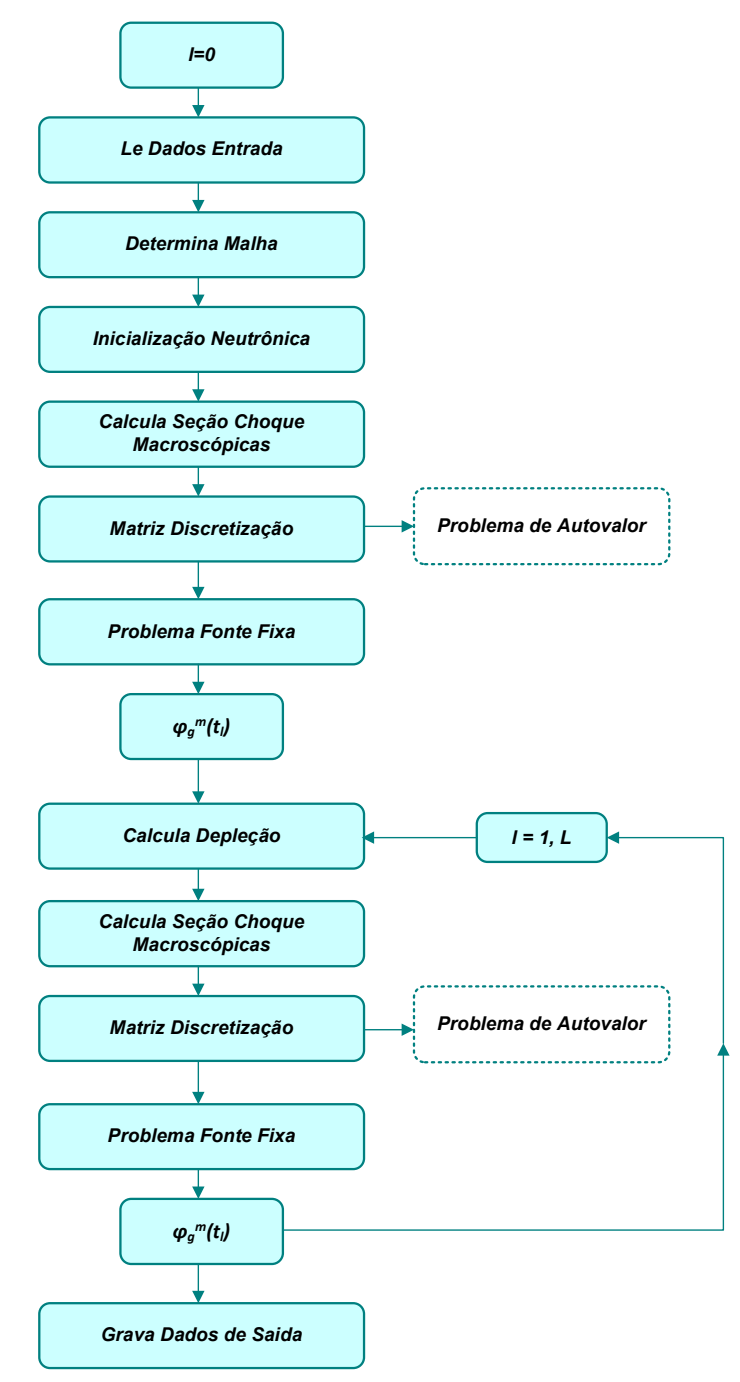

Figure 3: Flowchart of the Calculation of Transmutation

\section{Results}

The results with the "Calculation of Transmutation" program are shown in Table 1, which lists the residual concentration of MA irradiated during 300 days. The percentage of transmutation is 
obtained as follows: It can be observed that after 300 days, the rates of transmutation of Np237,

\begin{tabular}{c|c|c|c}
\hline \hline MA & $\begin{array}{c}\text { Initial Concentration } \\
(\text { atoms } / \text { b.cm })\end{array}$ & $\begin{array}{c}\text { Residual Concentration } \\
(\text { atoms } / \text { b.cm })\end{array}$ & Percentage of transmutation \\
\hline \hline $\mathrm{Np} 237$ & $1.390 \mathrm{E}-06$ & $1.38725 \mathrm{E}-06$ & $0.19 \%$ \\
\hline $\mathrm{Am} 241$ & $8.775 \mathrm{E}-08$ & $8.70233 \mathrm{E}-08$ & $0.83 \%$ \\
\hline $\mathrm{A} 242 \mathrm{~m}$ & $1.112 \mathrm{E}-09$ & $1.17626 \mathrm{E}-09$ & $-5.78 \%$ \\
\hline $\mathrm{Am} 242$ & $3.041 \mathrm{E}-10$ & $2.16087 \mathrm{E}-12$ & $99.3 \%$ \\
\hline $\mathrm{Am} 243$ & $2.041 \mathrm{E}-07$ & $2.03886 \mathrm{E}-07$ & $0.10 \%$ \\
\hline $\mathrm{Cm} 242$ & $3.338 \mathrm{E}-08$ & $9.68585 \mathrm{E}-09$ & $70.9 \%$ \\
\hline $\mathrm{Cm} 244$ & $3.534 \mathrm{E}-08$ & $3.44614 \mathrm{E}-08$ & $2.49 \%$ \\
\hline \hline
\end{tabular}

Table 1: Percentage of MA transmutation after 300 days of exposure.

Am241, Am242, Am243, Cm242 and Cm244 are 0.19\%, $0.83 \%, 99.3 \% 0.10 \%, 70.9 \%$ and 2.49 $\%$ respectively. These percentages show how these nuclides disappears. In contrast the percentage of nuclide Am $242 \mathrm{~m}$ was negative, indicating that the increased concentration of this nuclide.

The table 2 shows the values of $k_{\text {eff }}$ according the MA concentrations will be varied. Thus, this

\begin{tabular}{c|c|c|c|c|c}
\hline \hline $\begin{array}{c}\text { Fuel } \\
\text { (without BP) }\end{array}$ & $\begin{array}{c}\text { Fuel } \\
\text { (with BP) }\end{array}$ & $\begin{array}{c}\text { MA } \\
0.0243 \%\end{array}$ & $\begin{array}{c}\text { MA } \\
0.243 \%\end{array}$ & $\begin{array}{c}\text { MA } \\
2.43 \%\end{array}$ & $\begin{array}{c}\text { MA } \\
4.86 \%\end{array}$ \\
\hline 1.17939 & 0.96936 & 0.96870 & 0.96412 & 0.95115 & 0.94711 \\
\hline \hline
\end{tabular}

Table 2: Variation of $k_{e f f}$.

table shows the effective multiplication factors obtained with the 'Problema de Autovalor' subroutine, for different core loadings. The first column has the $k_{\text {eff }}$ of the fuel region without Burnable Poison (BP) and the transmutation region containing only water and structural material. In this case, it verifies that the reactor is super-critical. The second column presents the $k_{\text {eff }}$ for the fuel region now with BP, and the transmutation region is maintained without MA. From the third to sixth columns are shown the multiplication factors oF the fuel region with BP and now containing MA. The percentages that appear here were obtained through the ratio between the sum of the MA concentrations and the sum of the concentrations of nuclides in the fuel region. It can be seen that according MA concentrations increases, the $k_{\text {eff }}$ becomes smaller, i.e. MA can act like BP [7]. The results presented in the Table 1 are related to the third column of the table 2.

\section{Conclusions}

The development of this paper had as main issue the calculation of Minor Actinide transmutation in ADS reactors, for it was implemented an evolutive process called "Calculation of Transmutation". Firstly, the burning interval was taken of 300 days, which was in turn divided into time intervals of burning. For this purpose are created mainly two subroutine, one developed the temporary part and another the spatial part of the evolutive process. The first subroutine called "Calcula Depleção" is responsable to solve depletion equations at each instant in time. The second subroutine called "Problema de Fonte Fixa" was responsable for solving the neutron diffusion equation 
with a external neutron source. Also, it was observed that the effective multiplication factor increases with the evolution of time, due to MA are being transmuted, thus fissile materials are being created, all this happens due to permanent incineration of radioactive nuclides with an external source that does not stop, unless the provider of external neutron source is turned off. Additionally, it was seen that this study worked with the ratio of the sum of MA concentrations and the sum of the concentrations of nuclides in the fuel region equal to $0.0243 \%$. In this study still seen how is modified the effective multiplication factor according MA concentrations was varied, this was done in order to observe the behavior of subcriticality.

Is noticed that increasing of the MA concentrations in the transmutation region was decreasing the multiplication factor in the nuclear model, thus it is concluded that AM may act as absorbing materials such as BP. Finally, this work could be extended in a three-dimensional form and to take a cylindrical or annular geometry, typical of the configurations of the advanced reactors.

\section{References}

[1] Nuclear Technology Review 2013, IAEA, IAEA/NTR/2013, Viena, August 2013.

[2] IAEA nuclear energy series, Status of minor actinide fuel development, NF-T-4.6, International Atomic Energy Agency, Viena, 2009.

[3] Denis E. Beller and Gregory J. Van Tuyle and D. Bennett, The U.S. accelerator transmutation of waste program, Nuclear Instruments and Methods in Physics Research A, 463, 468-486, 2001 .

[4] Charles D. Bowman, Accelerator-driven Systems for nuclear waste transmutation, Annual Review of Nuclear and Particle Science, v. 48, pp. 505-556, 1998.

[5] Hamid A. Abderrahim and P. Baeten, A Multi-purpose hYbrid Research Reactor for High-tech Applications, Advances in Reactor Physics - Linking Research, Industry, and Education, 2012.

[6] A. C. M. Alvim and F. C. da Silva and A. S. Martinez, Depletion calculation for a nodal reactor physics code, Proceedings of the 18th International Conference on Nuclear Engineering, Xi'an, China, May,2010.

[7] B. Liu and $\mathrm{K}$. Wang and J. Tu, Transmutation of minor actinides in the pressurized water reactors, Annals of Nuclear Energy, v. 64, pp. 86-92, 2014. 